E2-2009-40

M. V. Tokarev ${ }^{1}$, I. Zborovský ${ }^{2}$

\title{
SELF-SIMILARITY OF PION PRODUCTION IN $A A$ COLLISIONS AT RHIC
}

Submitted to the XXXIX International Symposium on Multiparticle Dynamics, September 7-11, 2009, Belarus

\footnotetext{
${ }^{1}$ E-mail: tokarev@ sunhe.jinr.ru

${ }^{2}$ Nuclear Physics Institute, Academy of Sciences of the Czech Republic, Řež, Czech Republic; E-mail: zborovsky@ujf.cas.cz
} 
Токарев М.В., Зборовский И.

Самоподобие рождения пионов в $A A$-взаимодействиях на RHIC

Проведен анализ экспериментальных данных по инклюзивным сечениям рождения пионов в релятивистских столкновениях тяжелых ионов на RHIC в рамках $z$-скейлинга. Показано, что свойство самоподобия является характерной особенностью рождения пионов при столкновениях ядер высоких энергий. Обосновывается связь самоподобия со структурой сталкивающихся ядер, взаимодействием их конституентов и механизмами процесса фрагментации. Предложен микроскопический сценарий взаимодействия ядер на уровне конституентов. Исследована зависимость формы функции $\psi(z)$ и фрактальной размерности $\epsilon_{A A}$ от центральности столкновения. Получены оценки потерь энергии пионов в среде в зависимости от его поперечного импульса, энергии и центральности столкновения ядер. Обсуждается зависимость величины потерь от масштаба, на котором происходит взаимодействие конституентов. Установлено уменьшение «удельной теплоемкости» ядерной среды с ростом атомного номера ядра. Полученные результаты могут быть использованы для поиска и изучения новых явлений в процессах с рождением пионов в $p p$ - и $A A$-столкновениях высоких энергий при больших множественностях.

Работа выполнена в Лаборатории физики высоких энергий им.В.И.Векслера и А. М. Балдина ОИЯИ.

Препринт Объединенного института ядерных исследований. Дубна, 2009

Tokarev M. V., Zborovský I.

E2-2009-40

Self-Similarity of Pion Production in $A A$ Collisions at RHIC

Experimental data on inclusive spectra of pions produced in heavy ion collisions at RHIC are analyzed in the framework of $z$-scaling. The data indicate similarity as a characteristic feature of mechanism of pion production at high energies. It is argued that this property includes structure of the colliding objects, interaction of their constituents and mechanisms of the fragmentation process. A microscopic scenario of nucleus interactions at a constituent level in terms of momentum fractions is developed. The centrality dependence of the shape of the scaling function $\psi(z)$ and the fractal dimension $\epsilon_{A A}$ of the fragmentation process is studied. Energy losses of particles in the final state as a function of the collision energy, transverse momentum and centrality are estimated. The scale dependence of the energy losses is discussed. A decreasing tendency of specific heat of the produced medium with the system size is established. The obtained results may be exploited to search for and study of new physics phenomena in pion production in $p p$ and $A A$ collisions at high multiplicities.

The investigation has been performed at the Veksler and Baldin Laboratory of High Energy Physics, JINR. 


\section{INTRODUCTION}

The measurements of particle spectra at the Relativistic Heavy Ion Collider (RHIC) led to the discovery of a substantial suppression of hadron yields in nucleus-nucleus collisions relative to proton-proton data [1-4]. The suppression is observed in the region of high transverse momenta, typically more than few $\mathrm{GeV} / c$. It is connected with the energy radiations of the outgoing high- $p_{T}$ partons propagating through dense matter formed in the central collisions of heavy nuclei. The energy losses in the dense medium are substantially larger than in the vacuum. Quantification of the effect in the final state is a difficult problem depending on complicated calculations and model assumptions.

In the paper we analyze spectra of pions produced in heavy ion collisions at RHIC and develop a microscopic scenario of the spectra suppression in the framework of data $z$-presentation. The approach was applied for analysis of hadron production in $p p$ and $\bar{p} p$ collisions at high energies [5]. Above systems represent collisions of extended objects interacting in terms of their constituents. Production of particles from the constituent interactions is governed by the principles of self-similarity, locality, and fractality. The self-similarity of hadron production is valid both in soft and hard physics [6]. The locality and fractality are applied to the hard processes at small scales. The principles are manifested by the $z$-scaling observed in the production of charged and identified hadrons in $p p$ and $\bar{p} p$ collisions. The scaling represents independence of the scaling function $\psi(z)$ on the collision energy, types of the inclusive hadrons, their production angles, and includes spectra for various selection criteria with different charged multiplicities $N_{\text {ch }}$ [7]. The general principles can be applied to the nucleus-nucleus interactions as well. Here we demonstrate that pion spectra for different centrality classes in $A A$ collisions characterized by different multiplicity densities $d N_{\mathrm{ch}} / d \eta$ exhibit similar scaling behavior as in $p p$ collisions. This holds in a wide range of the transverse momentum $p_{T}$ and the collision energy $\sqrt{s}$. The shape of the function $\psi(z)$ for $A A$ systems depends on the collision centrality and differs from the scaling function for $p p$ interactions at high $z$. In the considered case of pions, a linear dependence of the fractal dimension $\epsilon_{A A}$ of the fragmentation process on the multiplicity density $d N_{\mathrm{ch}} / d \eta$ allows us to restore the centrality 
independent shape of $\psi(z)$ for $A A$ collisions which is identical with the $z$-scaling in $p p$ interactions. The increase of $\epsilon_{A A}$ with the multiplicity density is connected with larger energy losses of high- $p_{T}$ particles in the collisions of heavy nuclei. The region of small $p_{T}$ is characterized by low values of the scaling variable $z$. The single parameter $c$ which controls the behavior of $\psi(z)$ at low $z$ is interpreted as a «specific heat» of the produced medium. Performed analysis shows that it is independent of collision centrality but depends on the type of the colliding nuclei. Search for a possible change in the parameter $c$ is of interest especially for soft processes with high multiplicities. Such a change could be an indication of a phase transition in the matter produced in high energy collisions of both hadrons and nuclei.

The paper is organized as follows. A concept of the $z$-scaling and the method of construction of the scaling function $\psi(z)$ for both hadron and nucleus collisions are briefly described in Sec. 2. The properties of $\psi(z)$ for pion production in $p p$ collisions are mentioned in Sec.3. Results of analysis of pion spectra measured in $A A$ collisions at $\mathrm{RHIC}$ and $\mathrm{SpS}$ are presented in Sec.4. Pion production in $d A u$ collisions is studied in Sec.5. A microscopic scenario of the elementary subprocesses in $A A$ collisions is discussed and energy losses of the secondary particles are estimated in Sec.6. Conclusions are summarized in Sec. 7.

\section{INCLUSIVE SPECTRA IN $z$ PRESENTATION}

In this paper we follow the version of the $z$-scaling presented in $[5,6]$. Let us briefly remind the basic ideas of this concept. It is assumed that the collision of extended objects like hadrons and nuclei at sufficiently high energies is considered as an ensemble of individual interactions of their constituents. The constituents are partons in the parton model or quarks and gluons in the theory of QCD. A single interaction of constituents is illustrated in Fig. 1.

Structures of the colliding objects are characterized by parameters $\delta_{1}$ and $\delta_{2}$. The constituents of the incoming objects (hadrons or nuclei) with masses $M_{1}, M_{2}$ and momenta $P_{1}, P_{2}$ carry their fractions $x_{1}, x_{2}$. The inclusive particle carries the momentum fraction $y_{a}$ of the scattered constituent with a fragmentation characterized by a parameter $\epsilon_{a}$. A fragmentation of the recoil constituent is described by the parameter $\epsilon_{b}$ and the momentum fraction $y_{b}$. Multiple interactions of constituents are considered to be similar. This property reflects a self-similarity of the hadronic interactions at the constituent level.

2.1. Momentum Fractions $x_{1}, x_{2}, y_{a}$, and $y_{b}$. The idea of the $z$-scaling is based on the assumption [8] that gross features of an inclusive particle distribution of the reaction

$$
M_{1}+M_{2} \rightarrow m_{1}+X
$$




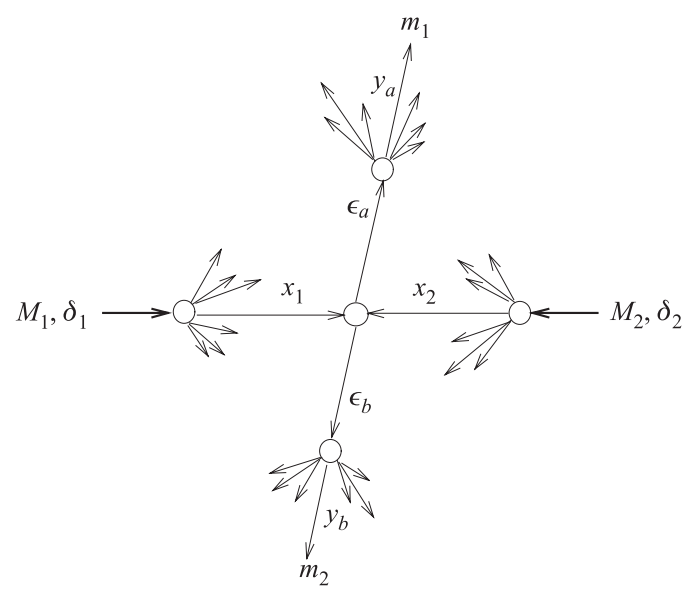

Fig. 1. Diagram of the constituent subprocess

can be described at high energies in terms of the kinematical characteristics of the corresponding constituent subprocess. We consider the subprocess to be a binary collision

$$
\left(x_{1} M_{1}\right)+\left(x_{2} M_{2}\right) \rightarrow\left(m_{1} / y_{a}\right)+\left(x_{1} M_{1}+x_{2} M_{2}+m_{2} / y_{b}\right)
$$

of the constituents $\left(x_{1} M_{1}\right)$ and $\left(x_{2} M_{2}\right)$ resulting in the scattered $\left(m_{1} / y_{a}\right)$ and recoil $\left(x_{1} M_{1}+x_{2} M_{2}+m_{2} / y_{b}\right)$ objects in the final state. The produced secondary objects transform into real particles after the constituent collisions. The registered particle with the mass $m_{1}$ and the 4-momentum $p$ and its hadron counterpart, moving in the opposite direction, carry the momentum fractions $y_{a}$ and $y_{b}$ of the scattered and recoil systems, respectively. The momentum conservation law of the constituent subprocess is connected with a recoil mass $M_{X}$ which we write in the form

$$
\left(x_{1} P_{1}+x_{2} P_{2}-p / y_{a}\right)^{2}=M_{X}^{2},
$$

where $M_{X}=x_{1} M_{1}+x_{2} M_{2}+m_{2} / y_{b}$. The associate production of $\left(m_{2}\right)$ ensures conservation of the additive quantum numbers. Equation (3) is an expression of the locality of the hadron interaction at a constituent level. It represents a kinematical constraint on the momentum fractions $x_{1}, x_{2}, y_{a}$, and $y_{b}$ which determine a subprocess (2).

Structure of the colliding objects and fragmentation of the systems formed in the scattered and recoil directions are characterized by the parameters $\delta_{1}, \delta_{2}$ and $\epsilon_{a}, \epsilon_{b}$, respectively. We connect the structural parameters with the corresponding 
momentum fractions by the function

$$
\Omega\left(x_{1}, x_{2}, y_{a}, y_{b}\right)=\left(1-x_{1}\right)^{\delta_{1}}\left(1-x_{2}\right)^{\delta_{2}}\left(1-y_{a}\right)^{\epsilon_{a}}\left(1-y_{b}\right)^{\epsilon_{b}} .
$$

Physical interpretation of $\Omega$ is given by its proportionality to relative number of all such constituent configurations in reaction (1) which contain the configuration defined by the fractions $x_{1}, x_{2}, y_{a}$, and $y_{b}$. The function $\Omega$ plays the role of a relative volume which occupy these configurations in the space of the momentum fractions. It was found that the structural parameters $\delta_{1}$ and $\delta_{2}$ have constant values at high energies. This holds for $\epsilon_{a}$ and $\epsilon_{b}$ in $p p$ collisions as well. The parameters are interpreted as fractal dimensions in the corresponding space of the momentum fractions. For proton-proton collisions we set $\delta_{1}=\delta_{2} \equiv \delta$. In the case of nucleus-nucleus collisions there are relations $\delta_{1}=A_{1} \delta$ and $\delta_{2}=A_{2} \delta$, where $A_{1}, A_{2}$ are atomic numbers. We assume that the fragmentation of the objects moving in the scattered and recoil directions can be described by the same parameter $\epsilon_{a}=\epsilon_{b} \equiv \epsilon_{F}$ which depends on the type $(F)$ of the inclusive particle. For given values of $\delta$ and $\epsilon_{F}$, we determine the fractions $x_{1}, x_{2}, y_{a}$, and $y_{b}$ in a way to maximize the function $\Omega\left(x_{1}, x_{2}, y_{a}, y_{b}\right)$, simultaneously fulfilling condition (3). The momentum fractions $x_{1}$ and $x_{2}$ obtained in this way can be decomposed as follows:

$$
x_{1}=\lambda_{1}+\chi_{1}, \quad x_{2}=\lambda_{2}+\chi_{2},
$$

where $\lambda_{1,2}=\lambda_{1,2}\left(y_{a}, y_{b}\right)$ and $\chi_{1,2}=\chi_{1,2}\left(y_{a}, y_{b}\right)$ are specific functions [5] of $y_{a}$ and $y_{b}$. Using the decomposition, the subprocess (2) can be rewritten into a symbolic form

$$
x_{1}+x_{2} \rightarrow\left(\lambda_{1}+\lambda_{2}\right)+\left(\chi_{1}+\chi_{2}\right) .
$$

This relation means that the $\lambda$ parts of the interacting constituents contribute to the production of the inclusive particle, while the $\chi$ parts are responsible for the creation of its recoil.

Since the momentum fractions are determined by means of the maximization of expression (4), they implicitly depend on $\delta$ and $\epsilon_{F}$. The parameter $\epsilon_{F}$ takes effectively into account also prompt resonances out of which the inclusive particle of a given type may be created. At fixed mass parameter $m_{2}$, larger values of $\epsilon_{F}$ correspond to smaller $y_{a}$ and $y_{b}$, which in turn give larger ratios $m_{2} / y_{b}$ and $m_{1} / y_{a}$. In our phenomenological approach this means that production of the inclusive particle $\left(m_{1}\right)$ and its counterpart $\left(m_{2}\right)$ is a result of fragmentation from larger masses which mimic in a sense processes with prompt resonances. Values of these parameters are determined in accordance with self-similarity requirements and experiment. In particular, this gives the restriction $m_{2}=m_{1}$.

2.2. Scaling Variable $z$ and Scaling Function $\psi(z)$. The self-similarity of hadron interactions reflects a property that hadron constituents and their interactions are similar. This is connected with dropping of certain dimensional 
quantities out of the description of physical phenomena. The self-similar solutions are constructed in terms of the self-similarity parameters. We search for a solution

$$
\psi(z)=\frac{1}{N \sigma_{\text {inel }}} \frac{d \sigma}{d z}
$$

depending on a single self-similarity variable $z$. Here $\sigma_{\text {inel }}$ is an inelastic cross section of reaction (1) and $N$ is an average particle multiplicity. The variable $z$ is defined as follows:

$$
z=z_{0} \Omega^{-1}
$$

where

$$
z_{0}=\frac{\sqrt{s_{\perp}}}{\left(d N_{\mathrm{ch}} /\left.d \eta\right|_{0}\right)^{c} m}
$$

and $\Omega$ given by (4). For given reaction (1), $z$ is proportional to the transverse kinetic energy $\sqrt{s_{\perp}}$ of the constituent subprocess (2) consumed on the production of the inclusive particle $\left(m_{1}\right)$ and its counterpart $\left(m_{2}\right)$. The energy $\sqrt{s_{\perp}}$ is determined by the formula

$$
\sqrt{s_{\perp}}=T_{a}+T_{b}
$$

where

$$
\begin{aligned}
& T_{a}=y_{a}\left(\sqrt{s_{\lambda}}-M_{1} \lambda_{1}-M_{2} \lambda_{2}\right)-m_{1}, \\
& T_{b}=y_{b}\left(\sqrt{s_{\chi}}-M_{1} \chi_{1}-M_{2} \chi_{2}\right)-m_{2} .
\end{aligned}
$$

The terms

$$
\sqrt{s_{\lambda}}=\left[\left(\lambda_{1} P_{1}+\lambda_{2} P_{2}\right)^{2}\right]^{1 / 2}, \quad \sqrt{s_{\chi}}=\left[\left(\chi_{1} P_{1}+\chi_{2} P_{2}\right)^{2}\right]^{1 / 2}
$$

represent the energy for production of the secondary objects moving in the scattered and recoil directions, respectively. The quantity $d N_{\mathrm{ch}} /\left.d \eta\right|_{0}$ is the corresponding multiplicity density of charged particles in the central region of reaction (1) at pseudorapidity $\eta=0$. The multiplicity density in the central interaction region is related to a state of the produced medium. The parameter $c$ characterizes properties of this medium [5]. It is determined from multiplicity dependence of inclusive spectra [5-7]. The mass constant $m$ is arbitrary and we fix it at the value of nucleon mass.

The scaling function $\psi(z)$ is expressed in terms of the experimentally measured inclusive cross section $E d^{3} \sigma / d p^{3}$, the multiplicity density $d N / d \eta$ at pseudorapidity $\eta$, and $\sigma_{\text {inel }}$. Exploiting the definition (7) one can obtain the expression [5] 


$$
\psi(z)=-\frac{\pi s}{(d N / d \eta) \sigma_{\text {inel }}} J^{-1} E \frac{d^{3} \sigma}{d p^{3}},
$$

where $s$ is the square of the center-of-mass energy and $J$ is the corresponding Jacobian. The multiplicity density $d N / d \eta$ in expression (13) concerns particular hadrons species. It depends on the center-of-mass energy, on various multiplicity selection criteria, and also on the production angles at which the inclusive spectra were measured. The procedure of obtaining the corresponding values of $d N / d \eta$ from the $p_{T}$ spectra is described in [5]. The function $\psi(z)$ is normalized as follows:

$$
\int_{0}^{\infty} \psi(z) d z=1 .
$$

The above relation allows us to interpret the function $\psi(z)$ as a probability density to produce an inclusive particle with the corresponding value of the variable $z$.

\section{SELF-SIMILARITY OF PION PRODUCTION IN $p p$ COLLISIONS}

Let us remind main features of the $z$-scaling in proton-proton interactions characterized by a proton fractal dimension $\delta_{1}=\delta_{2} \equiv \delta$ in the initial state. Experimental data on inclusive cross sections of charged and identified hadrons measured in $p p$ collisions at FNAL, ISR, and RHIC have been analyzed in [5, 6]. The data cover a wide range of the collision energy, transverse momenta, and angles of the produced particles. Spectra from minimum-biased events and events with various multiplicity selection criteria have been studied in [7]. The energy, angular, and multiplicity independence of the scaling function $\psi(z)$ was established. It gives strong constraints on the values of the parameters $c, \delta$, and $\epsilon$. It was shown that the parameters are constant in large kinematical region. Non of the parameters depends on multiplicity. The scaling is consistent with $c=0.25$ and $\delta=0.5$ for all types of the analyzed inclusive hadrons. The value of $\epsilon$ increases with hadron mass [6].

Some results of the analysis are illustrated on the experimental data [912] depicted by different symbols in Fig. 2, $a$. The data cover large range of the collision energies $\sqrt{s}_{N N}=19-200 \mathrm{GeV}$ and transverse momenta $p_{T}=$ $0.2-10 \mathrm{GeV} / c$ of the produced hadrons. The distributions were measured in the central interaction region at $\theta_{\mathrm{cms}}=90^{\circ}$. The scaling function $\psi(z)$ is shown in Fig. $2, b$ where the charged hadron spectra at different energies are «collapsed» onto a single curve.

The behavior of $\psi(z)$ is described by the power law, $\psi(z) \sim z^{-\beta}$, in the asymptotic high- $z\left(\right.$ high- $\left.p_{T}\right)$ region. 

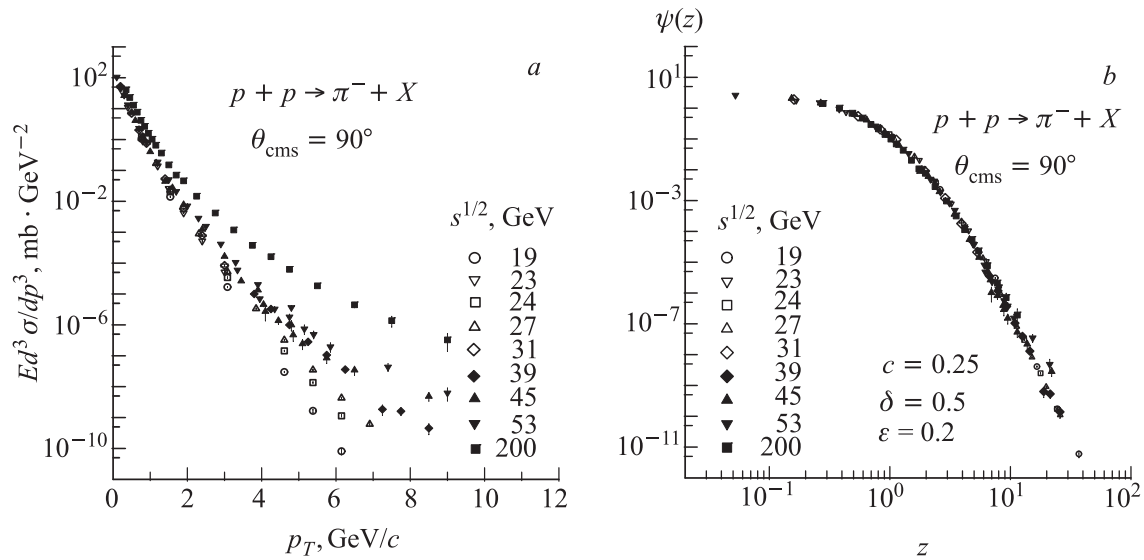

Fig. 2. a) Transverse momentum spectra of the $\pi^{-}$mesons produced in $p p$ collisions at $\sqrt{s}_{N N}=19-200 \mathrm{GeV}$. Experimental data are taken from [9-12]. b) The corresponding scaling function $\psi(z)$

\section{SELF-SIMILARITY OF PION PRODUCTION IN $A A$ COLLISIONS}

Here we present some ideas how to quantify energy losses of the produced particles exploiting a specific connection between the suppression of the inclusive spectra in nuclear collisions and the corresponding multiplicity densities in dependence on the collision centrality. The ideas are motivated by the assumption of self-similarity of hadron interactions at a constituent level both in $p p$ and $A A$ collisions. This is demonstrated on pion spectra obtained at RHIC and SpS.

The STAR Collaboration measured the spectra of negative pions [13] produced in AuAu collisions at $\sqrt{s_{N N}}=200 \mathrm{GeV}$. The spectra were taken at various centralities characterized by different multiplicity densities $d N_{\mathrm{ch}} /\left.d \eta\right|_{0}$ of charged particles produced in the central interaction region. They cover a wide range of the transverse momentum, $p_{T}=0.35-10.86 \mathrm{GeV} / c$. The yields (Fig. 3, $a$ ) change more than eight orders of magnitude in this range. The centrality dependence of the pion spectra is plotted in $z$-presentation in Fig. 3, $b$. The corresponding $p p$ data are represented by the solid line. The parameter $\epsilon \equiv \epsilon_{\pi}=0.2$ was fixed at the same value as for scaling analysis of pions in the $p p$ collisions. The fractal dimension $\delta_{\mathrm{Au}}$ of the colliding nuclei was determined in accordance with the additive property $\delta_{A}=A \delta$ obtained for $p A$ interactions [14] with the same value of $\delta=0.5$ as for $p p$ data. The corresponding total multiplicity densities $d N_{\mathrm{ch}} /\left.d \eta\right|_{0}$ of charged particles produced in AuAu collisions have been used in formula (9). They depend on the centrality of the nuclear collisions. In the case of $p p$ interactions, the multiplicity density $d N_{\mathrm{ch}} /\left.d \eta\right|_{0}$ for the non-single-diffractive $p p$ events was used in (9) for comparative reasons. 

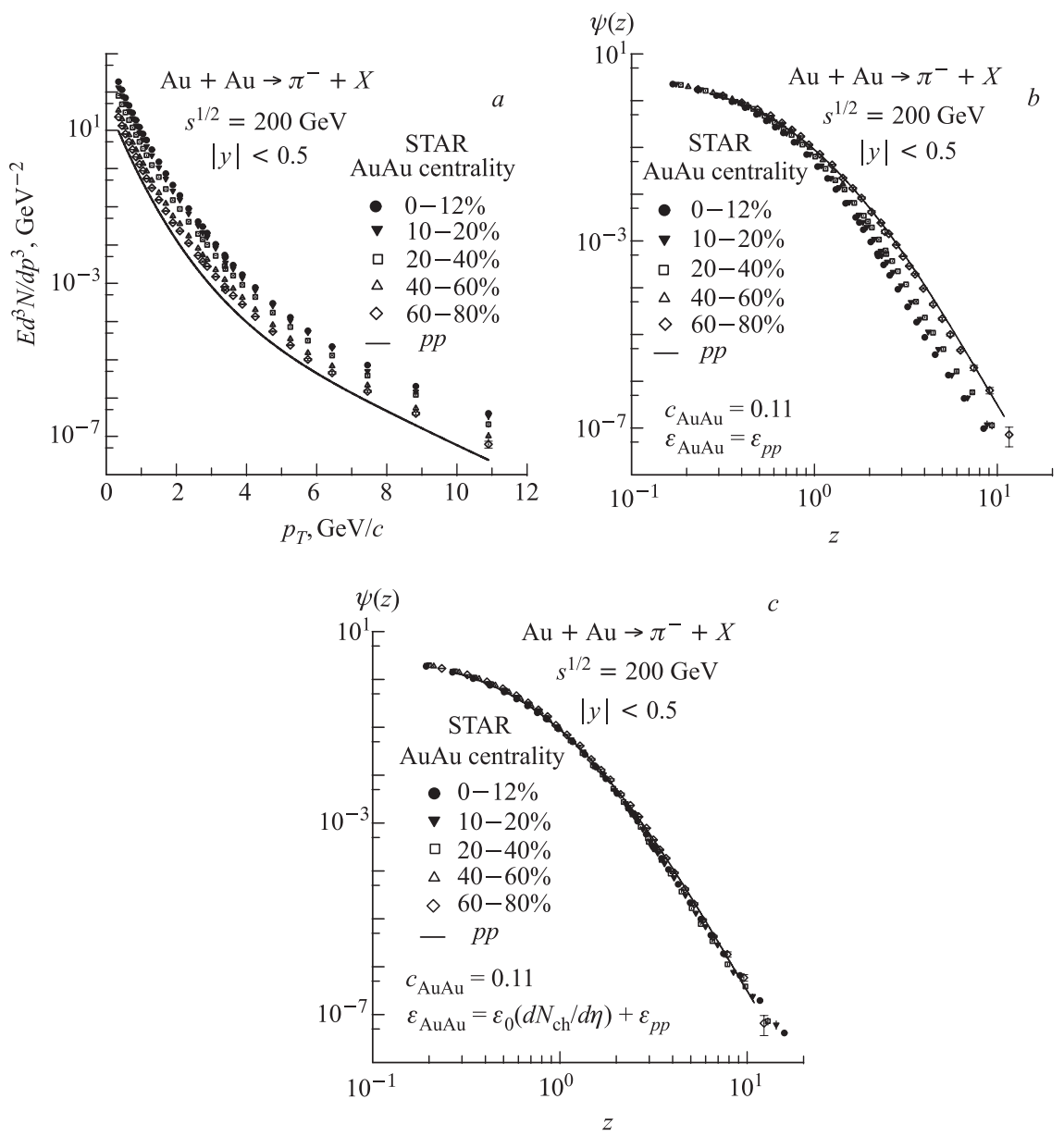

Fig. 3. The inclusive spectra of negative pions produced in $p p$ and AuAu collisions [13] for different centralities at $\sqrt{s_{N N}}=200 \mathrm{GeV}$ in $a$ ) $p_{T^{-}}$and $z$-presentation for $b$ ) constant and c) multiplicity-dependent parameter $\epsilon_{\mathrm{AuAu}}$. The solid lines represent the scaling function for $p p$ collisions

As seen from Fig. $3, b$, the spectra in $p p$ and peripheral AuAu collisions coincide each other with good accuracy in $z$-presentation for $c_{\mathrm{AuAu}}=0.11$. This value is consistent with the energy independence of $z$-presentation of the pion spectra in the peripheral AuAu collisions. The result indicates that the form of the pion spectra in peripheral collisions is unsensitive to modifications of the production mechanism by nuclear medium when compared with $p p$ interactions. 

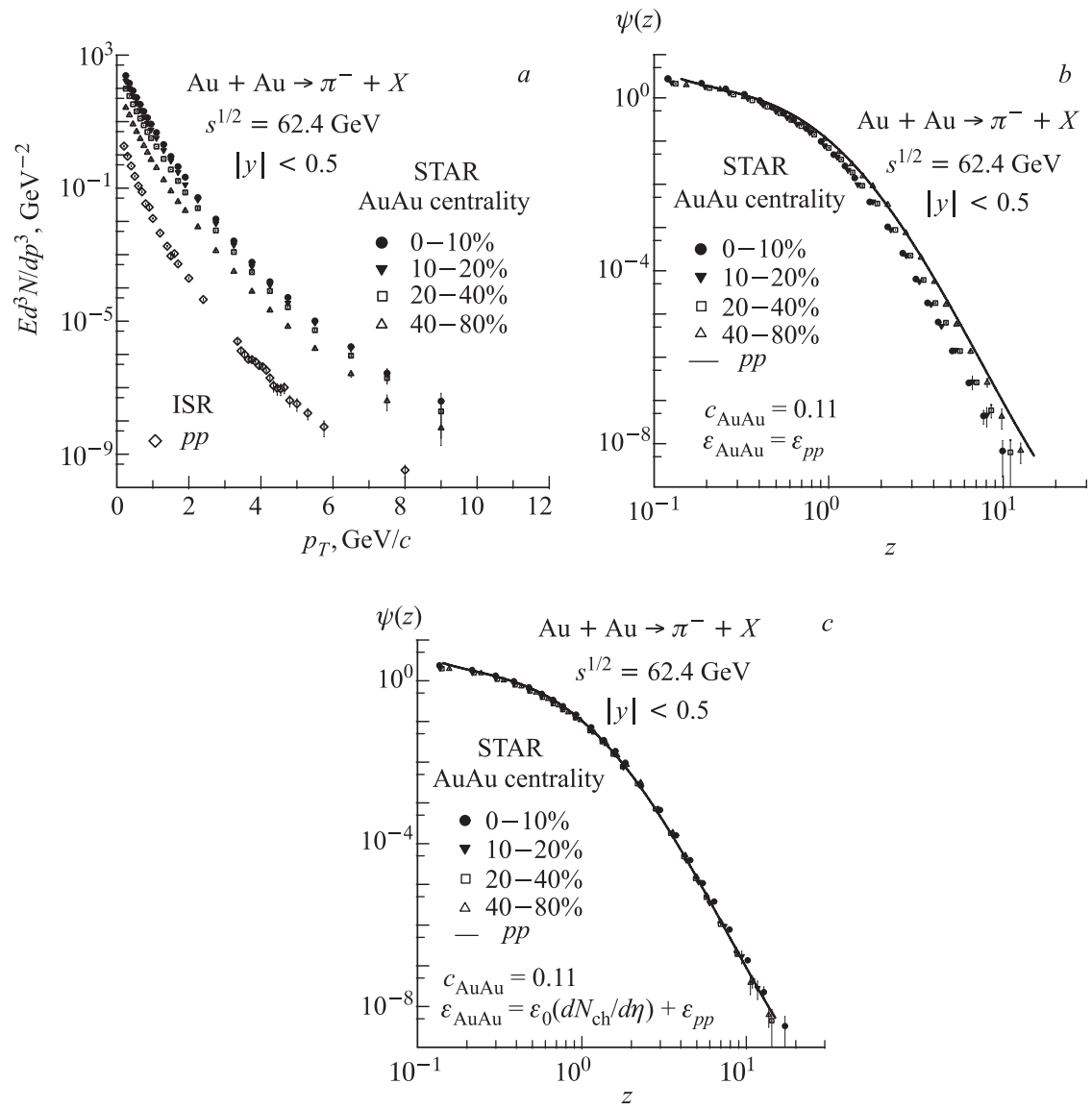

Fig. 4. The inclusive spectra of negative pions produced in $p p[19,20]$ and AuAu [15] collisions for different centralities at $\sqrt{s_{N N}}=62.4 \mathrm{GeV}$ in $a$ ) $p_{T^{-}}$- and $z$-presentation for b) constant and $c$ ) multiplicity-dependent parameter $\epsilon_{\mathrm{AuAu}}$. The solid lines represent the scaling function for $p p$ collisions

The influence of nuclei is included here with a drop-off in the «specific heat» $c$ from its value $c_{p p}=0.25$ obtained in $p p$ collisions. One can see from Fig. $3, b$ that the AuAu spectra are suppressed in the high- $z$ region relatively to the $p p$ scaling function $\psi(z)$ as the centrality increases. The largest suppression is for the most central collisions.

The same scaling behavior as for $p p$ collisions can be obtained for $\mathrm{AuAu}$ interactions for all centralities. As shown in Fig. 3, $c$, this can be achieved by the parameter $\epsilon_{A A}$ allowing it to be a function of the multiplicity density $d N_{\mathrm{ch}} / d \eta$. 

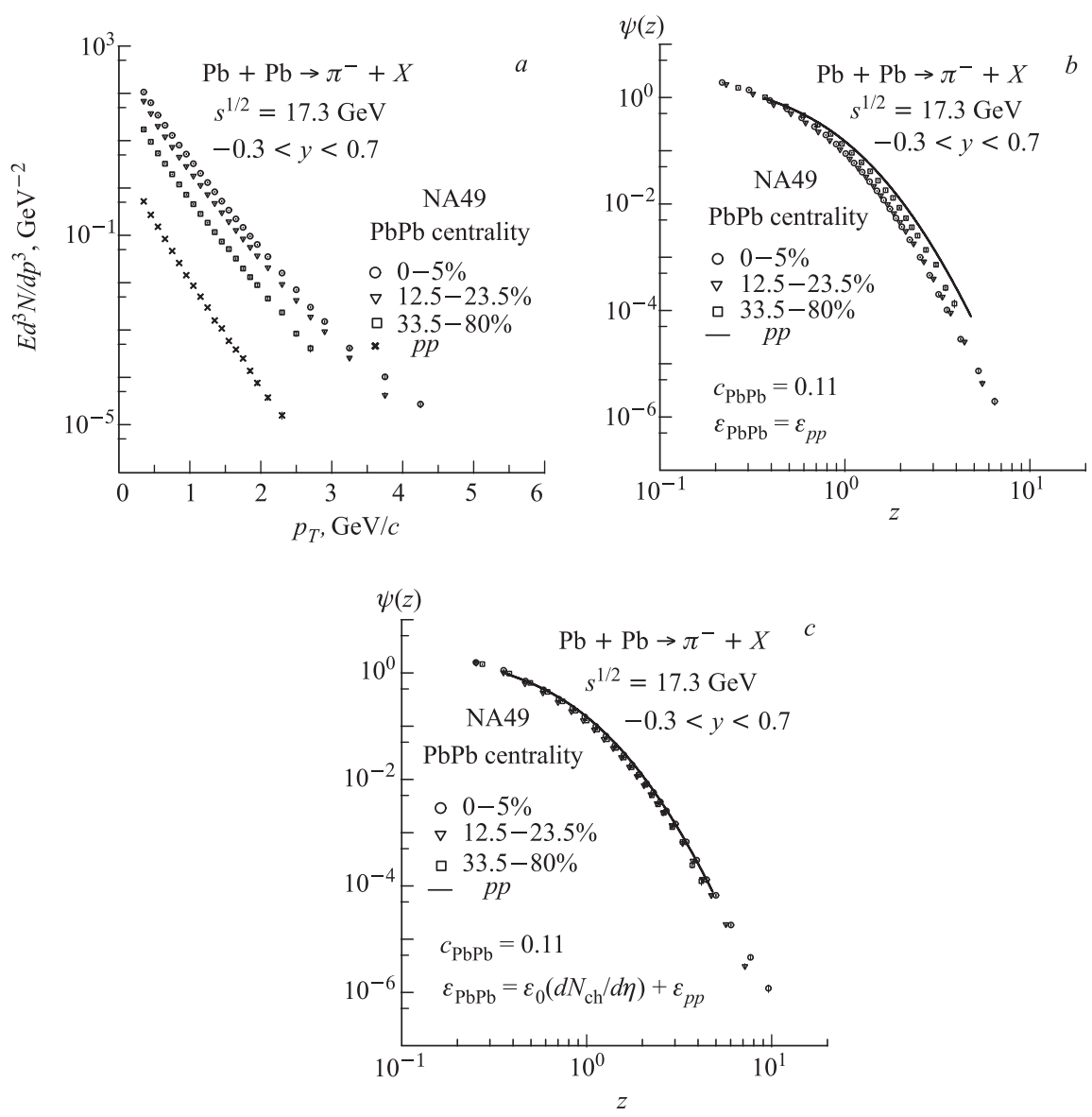

Fig. 5. The inclusive spectra of negative pions produced in $p p$ and $\mathrm{PbPb}$ collisions [16] for different centralities at $\sqrt{s_{N N}}=17.3 \mathrm{GeV}$ in $\left.a\right) p_{T}$ - and $z$-presentation for $b$ ) constant and c) multiplicity-dependent parameter $\epsilon_{\mathrm{PbPb}}$. The solid lines represent the scaling function for $p p$ collisions

For that purpose we have used the parametrization

$$
\epsilon_{A A}=\epsilon_{0}\left(d N_{\mathrm{ch}} / d \eta\right)+\epsilon_{p p}
$$

with a suitable choice of the coefficient $\epsilon_{0}$. For AuAu collisions at $\sqrt{s_{N N}}=$ $200 \mathrm{GeV}$ we obtained $\epsilon_{0}=0.0028$. The increase of $\epsilon$ with the multiplicity density is connected with a decrease of the momentum fractions $y_{a}$ and $y_{b}$. This results in larger energy losses in the final state. The energy losses depend on the 
amount of the traversed medium which converts them into the multiplicity of the produced particles. The larger $\epsilon$ the more energy losses of the secondary particles. The multiplicity density characterizes the produced medium and is connected in a such way to the energy losses in this medium. Results of similar analysis of the STAR data [15] on pion spectra at different centralities in AuAu collisions at $\sqrt{s_{N N}}=62.4 \mathrm{GeV}$ are shown in Fig. 4. We have obtained $\epsilon_{0}=0.0018$ in this case.

The NA49 Collaboration measured the transverse momentum spectra of negative pions [16] in $\mathrm{PbPb}$ collisions at $\sqrt{s_{N N}}=17.3 \mathrm{GeV}$. The invariant yields were extracted as a function of the transverse momentum $p_{T}$ in the range from 0.3 to $4.5 \mathrm{GeV} / c$ in the rapidity interval $-0.3<y<0.7$ at different collision centralities. Figure 5 demonstrates data on pion spectra with three centrality ranges in $p_{T^{-}}(a)$ and $z$-presentation without $(b)$ and with $(c)$ centrality dependence of $\epsilon_{\mathrm{PbPb}}$. As seen from Fig. 5, $b$ the shape of the function $\psi(z)$ at $\epsilon_{\mathrm{PbPb}}=\epsilon_{p p}$ depends on centrality. The uniform shape can be restored with $\epsilon_{0}=0.0010$ (15) and $c_{\mathrm{PbPb}}=0.11$. The solid line shown in Fig. 5, $c$ corresponds to $p p$ data at the same energy.

The STAR Collaboration measured for the first time the identified particle spectra in AuAu collisions at $\sqrt{s}_{N N}=9.2 \mathrm{GeV}$ [17]. The restricted data sample (3000 events) allows one to compare the hadron yields, particle ratios or $\left\langle p_{T}\right\rangle$ to the same quantities obtained from $\mathrm{SpS}$ experiments at similar beam energies.
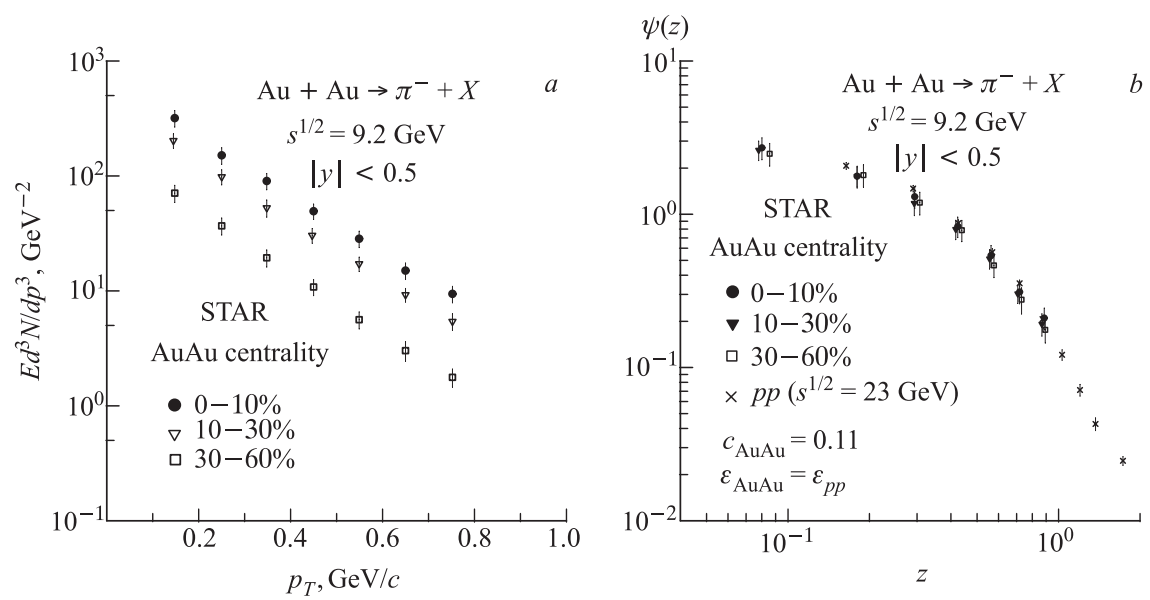

Fig. 6. The inclusive spectra of negative pions produced in AuAu collisions [17] for different centralities at $\sqrt{s}_{N N}=9.2 \mathrm{GeV}$ in $a$ ) $p_{T^{-}}$and $b$ ) $z$-presentation for constant parameter $\epsilon_{\mathrm{AuAu}}$ 
The transverse momentum distributions at different centralities cover the range $p_{T}=0.15-0.75 \mathrm{GeV} / c$ and $|\eta|<0.5$. The spectra of $\pi^{-}$mesons in $p_{T^{-}}$and $z$-presentations are shown in Fig. 6, $a$ and 6, $b$, respectively. As seen from Fig. 6, $b$ the experimental data points do not reveal any dependence of $\epsilon_{\mathrm{AuAu}}$ on centrality in this $p_{T}$-range. Sensitivity of the shape of $\psi(z)$ to the collision centrality is expected to be enhanced for $z>1$. A verification of the power law, $\psi(z) \sim z^{-\beta}$, of the scaling function for $z>4$ is of interest in this energy region as well. A self-similarity of hadron substructure, constituent interactions, and fragmentation processes is visible in the pion spectra at higher energies. Measurements of pion distributions at $\sqrt{s}_{N N}=9.2 \mathrm{GeV}$ in the range $p_{T}=2-3 \mathrm{GeV} / c$ is therefore desirable to study centrality dependence of the energy losses in this energy region.

\section{SELF-SIMILARITY OF PION PRODUCTION IN $d$ Au COLLISIONS}

We have analyzed data [18] on the transverse momentum distributions of pion spectra in $d \mathrm{Au}$ collisions at $\sqrt{s}_{N N}=200 \mathrm{GeV}$ and $|\eta|<0.5$. The pion yields measured over a range of $p_{T}=0.2-9.5 \mathrm{GeV} / c$ at different centralities are shown in $p_{T^{-}}$and $z$-presentations in Fig. 7. The fractal dimensions for deuteron and gold are $\delta_{d}=2 \delta$ and $\delta_{\mathrm{Au}}=197 \delta$, respectively. The «specific heat» of the produced matter in this process is found to be $c=0.23$, what is nearly the same as in proton-proton collisions $\left(c_{p p}=0.25\right)$. This value does not depend on kinematical parameters. Figure 7, $c$ shows that centrality independence of the shape of the function $\psi(z)$ can be restored (the same shape for $p p$ and $d \mathrm{Au}$ ) if the fractal dimension $\epsilon_{d \mathrm{Au}}$ of the fragmentation processes is assumed to be in the form (15). An indication of the power behavior of $\psi(z)$ is seen at high $z$. The values of the parameter $\epsilon_{0}$ depend on the system size and collision energy. We have obtained $\epsilon_{0}(d \mathrm{Au})=0.04>\epsilon_{0}(\mathrm{CuCu})=0.008>\epsilon_{0}(\mathrm{AuAu})=0.0028$ at $\sqrt{s}_{N N}=200 \mathrm{GeV}$. In this sense, the parameter $\epsilon_{0}$ does not represent a scaling universal quantity. Its energy and system size dependences characterize different energy losses discussed in the next section.

\section{ENERGY LOSSES IN TERMS OF MOMENTUM FRACTIONS}

In this section we use a microscopic scenario of particle production in terms of momentum fractions for estimation of the energy losses of the produced particles in nuclear medium. In the framework of such a scenario, any inclusive particle is characterized by the quantity $z$. It consists of the finite part $z_{0}$ and of the divergent factor $\Omega^{-1}$. The factor $\Omega^{-1}$ depends on the momentum fractions $x_{1}, x_{2}$ and $y_{a}, y_{b}$ which carry the incoming constituents and the inclusive particle $\left(m_{1}\right)$ with its counterpart $\left(m_{2}\right)$, respectively. The variable $z$ is the scale-dependent 

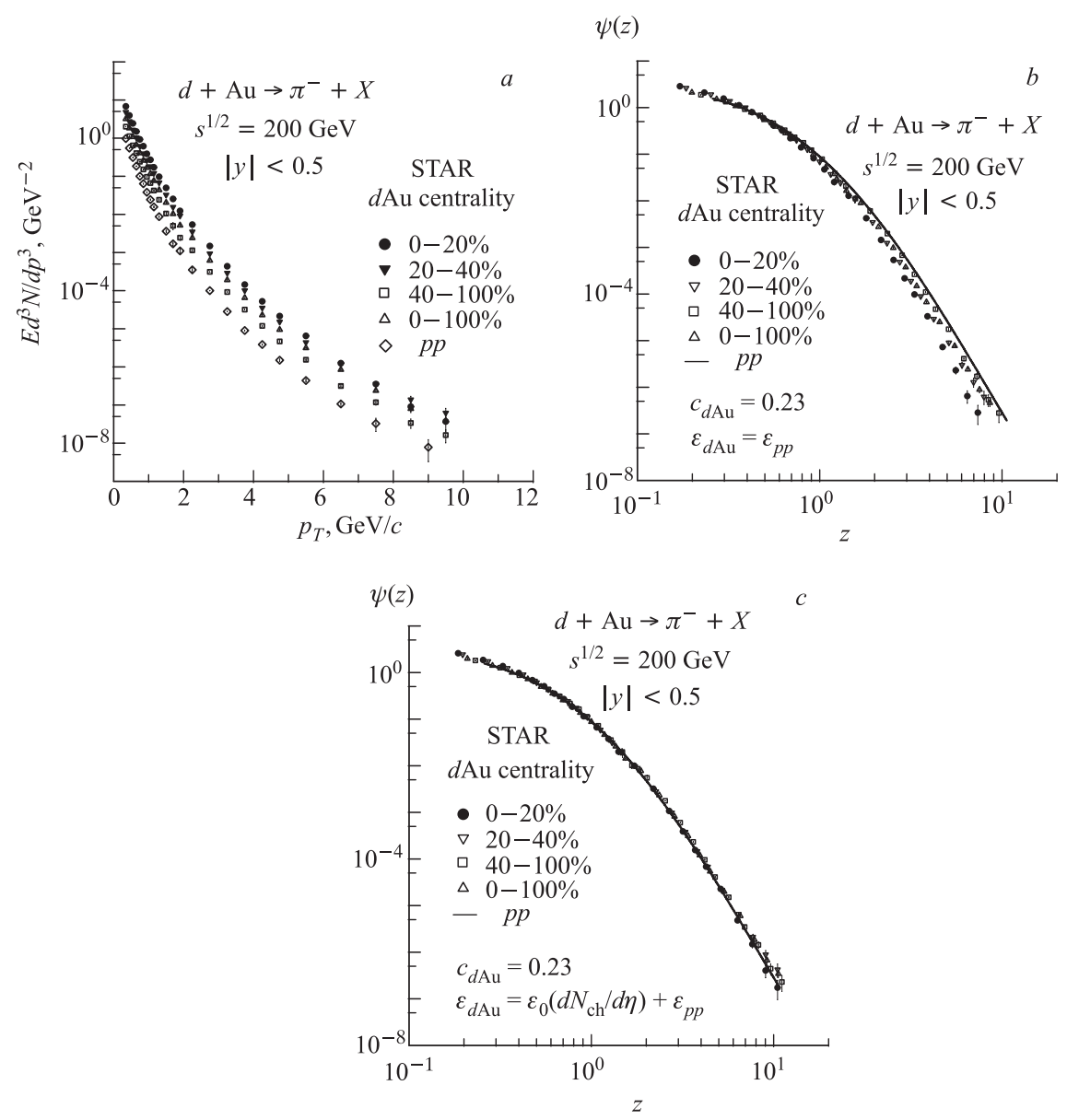

Fig. 7. The inclusive spectra of $\pi^{-}$pions produced in $d$ Au collisions [18] for different centralities at $\sqrt{s}_{N N}=200 \mathrm{GeV}$ in $a$ ) $p_{T^{-}}$and $z$-presentation for $b$ ) constant and c) multiplicity-dependent parameter $\epsilon_{d \mathrm{Au}}$. The solid lines represent the scaling function for $p p$ collisions

quantity. The principle of minimal resolution $\Omega^{-1}$ allows us to fix the values of the momentum fractions $x_{1}, x_{2}, y_{a}$, and $y_{b}$ and single out the most effective binary subprocess which underlies the inclusive reaction. The recoil mass $M_{X}$ characterizes dissipation of the recoil object moving in the away side of the inclusive particle. The quantities $y_{a}$ and $M_{X}$ are therefore important ingredients of the fragmentation process. Some dependences of the momentum fraction 

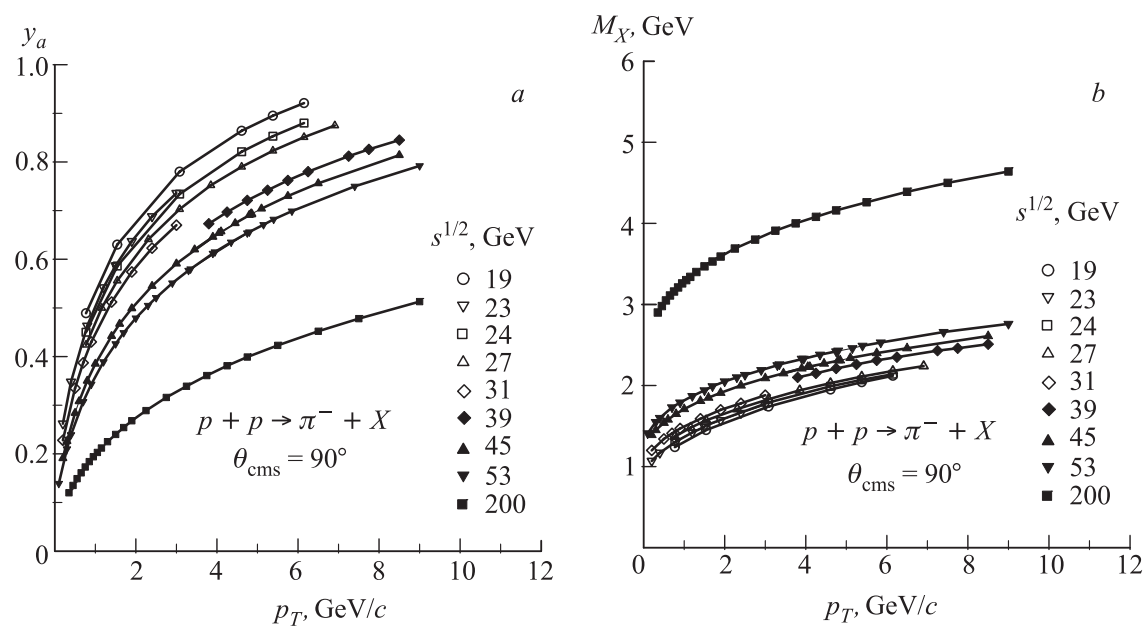

Fig. 8. The dependence of the fractions $y_{a}(a)$ and the recoil mass $M_{X}(b)$ on the transverse momentum $p_{T}$ for $\pi^{-}$mesons produced in $p p$ collisions at $\theta_{\mathrm{cms}}=90^{\circ}$ and different collision energies

$x_{1}, x_{2}, y_{a}, y_{b}$ and the recoil mass $M_{X}$ on the transverse momentum $p_{T}$ of $\pi^{-}$ mesons produced in $p p, \mathrm{AuAu}$, and $\mathrm{PbPb}$ collisions are shown in Figs. 8-12.

6.1. Momentum Fractions versus $p_{T}$. Study of the momentum fractions and their dependences on the collision energy, centrality, and transverse momentum of the inclusive particle gives us possibility to look at the microscopic picture of the underlaying subprocesses. The fractions $x_{1}$ and $x_{2}$ characterize amount of the energy (momentum) of the interacting nuclei carried by their constituents which undergo the binary collision (2). The fractions $y_{a}$ and $y_{b}$ characterize energy losses of the scattered and recoil constituents during their fragmentation in vacuum for $p p$ collisions or in medium in the case of nuclear interactions.

Figure 8 shows the dependence of the fraction $y_{a}(a)$ and the recoil mass $M_{X}(b)$ on the transverse momentum of pions produced in $p p$ collisions at $\sqrt{s}_{N N}=19-200 \mathrm{GeV}$ and $\theta_{\mathrm{cms}}=90^{\circ}$. As seen from Fig. 8, $a$ the energy loss characterized by a deviation of the fraction $y_{a}$ from unity decreases with the increasing momentum $p_{T}$ and increases with the collision energy $\sqrt{s}$. Let us consider a pion with the momentum $6 \mathrm{GeV} / c$ produced in $p p$ collisions. In the presented scenario, the pion is produced by fragmentation of a secondary parton with the momentum $p_{T}=6 / 0.9=6.6$ or $6 / 0.4=15 \mathrm{GeV} / c$ at $\sqrt{s}_{N N}=19$ or $200 \mathrm{GeV}$, respectively. This quantitative comparison characterizes increase of the energy losses with the collision energy. The recoil mass $M_{X}$ increases with both the energy and pion transverse momentum in a way shown in Fig. 8, $b$. 
Figures $9, a, 10, a, 11, a$, and 12, $a$ demonstrate the dependence of the momentum fraction $x_{1}$ expressed in units of nucleon mass on the transverse momentum $p_{T}$ of negative pions produced in $A A$ collisions at $\sqrt{s}_{N N}=200,62.4,17,3$, and $9.2 \mathrm{GeV}$, respectively. The points correspond to data at $\theta_{\mathrm{cms}} \simeq 90^{\circ}$. Both fractions $x_{1}$ and $x_{2}$ are equal to each other in this case. The fraction $x_{1}$ increases with the transverse momentum $p_{T}$ and centrality. For fixed $p_{T}, x_{1}$ decreases as the collision energy $\sqrt{s}$ increases.

Figures $9, b, 10, b, 11, b$, and $12, b$ show the dependences of the momentum fractions $y_{a}$ on the kinematical $\left(p_{T}, \sqrt{s}\right)$ and dynamical $\left(d N_{\mathrm{ch}} / d \eta\right)$ variables. They describe features of the fragmentation process. The fraction $y_{a}$ characterizes dissipation (energy loss) of the momentum of the object produced in the underlying constituent interaction into the near side of the inclusive particle. The behavior of $y_{a}$ demonstrates a monotonic growth with $p_{T}$. It means that the energy losses associated with the production of a high- $p_{T}$ pion are smaller than for the inclusive processes with lower transverse momenta. The decrease of $y_{a}$ with centrality in $\mathrm{AuAu}$ and $\mathrm{PbPb}$ collisions represents larger energy losses in the central collisions as compared with the $p p$ and peripheral $A A$ interactions. Energy dissipation grows as the collision energy increases. Let us consider for definiteness a pion produced in the central AuAu collisions $(0-12 \%)$ at $\sqrt{s}_{N N}=200 \mathrm{GeV}$ which has the momentum $p_{T}=4 \mathrm{GeV}$. The pion was formed in the fragmentation of the secondary parton produced in the constituent interaction with the momentum $p_{T}=4 / 0.1=40 \mathrm{GeV}$. The momenta of such partons decrease with the decreasing collision energy and are equal to $4 / 0.3 \simeq 13$ and $4 / 0.55 \simeq 7.3 \mathrm{GeV} / c$ at $\sqrt{s}_{N N}=62.4$ and $17.3 \mathrm{GeV}$, respectively. The fraction $y_{a}$ follows the general trend, i.e., a decrease with the collision energy and centrality, and a growth with the transverse momentum $p_{T}$.

The fraction $y_{b}$ governs the recoil mass $M_{X}$ characterizing the dissipation of the energy and momentum in the away side direction of the inclusive particle. It was found that $y_{b}$ is much smaller than $y_{a}$ at high $p_{T}$. The small values of $y_{b}$ mean that the momentum of the inclusive particle is more likely balanced by a multiple system consisting of many low momentum particles moving in the opposite direction. The fraction $y_{b}$ decreases with the increasing collision energy $\sqrt{s}$. Its dependence on $p_{T}$ and centrality is weak. Both fractions become equal to each other, $y_{a} \simeq y_{b}$, at low transverse momenta. The equality at low $p_{T}$ remains preserved independent on $\sqrt{s}$.

6.2. Mass of Recoil System versus $p_{T}$. The recoil mass $M_{X}$ of the system associated with production of the inclusive particle is defined by the expression

$$
M_{X}=x_{1} M_{1}+x_{2} M_{2}+m_{2} / y_{b}
$$

The quantity is proportional to the momentum fractions $x_{1}$ and $x_{2}$ of the interacting objects with the masses $M_{1}$ and $M_{2}$. It has connection with the fractions 

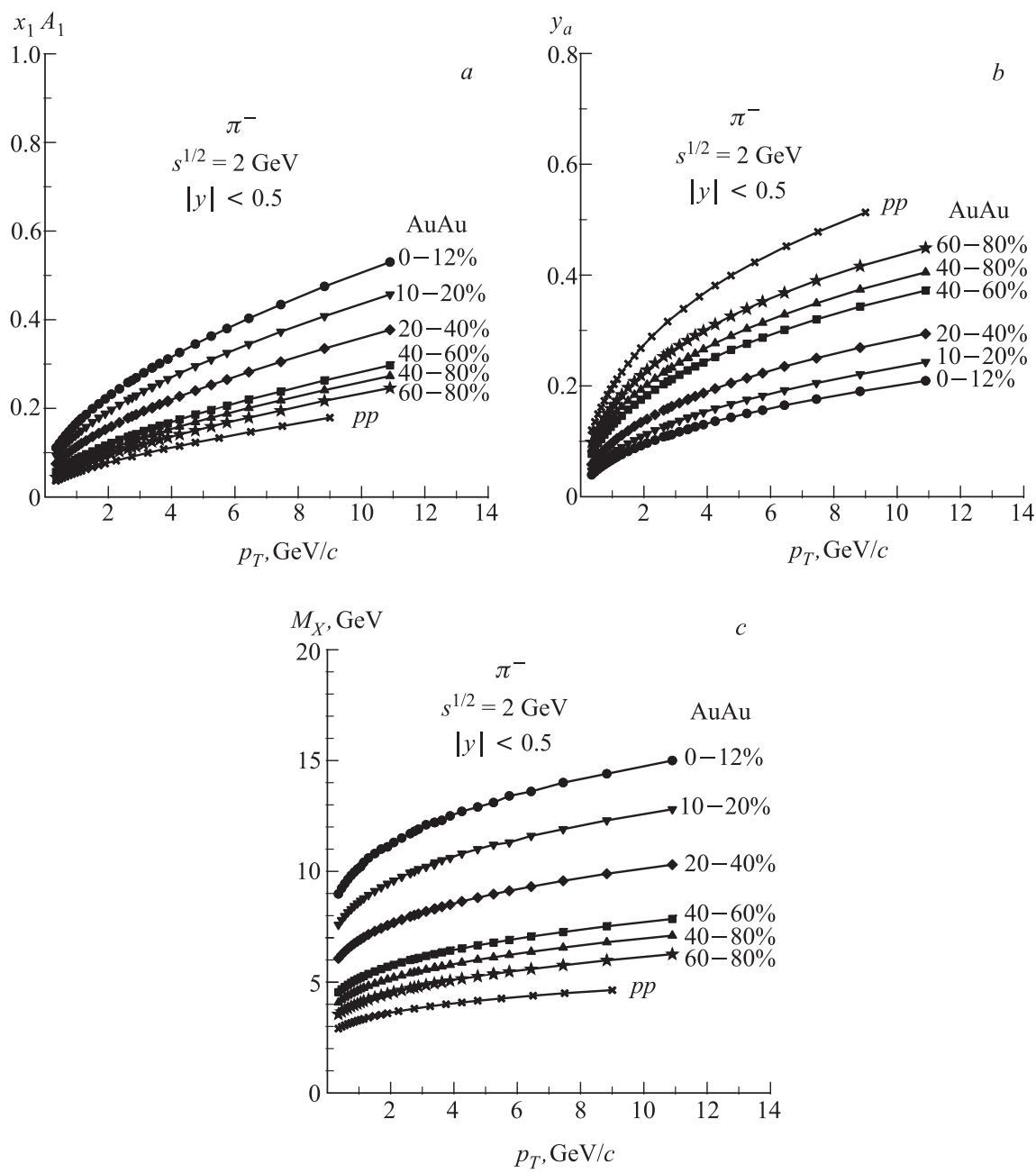

Fig. 9. The dependence of the fractions $A_{1} x_{1}(a), y_{a}(b)$, and the recoil mass $M_{X}(c)$ on the transverse momentum $p_{T}$ for $\pi^{-}$mesons produced in $p p$ and AuAu collisions at different centralities. The symbols correspond to data measured at $\sqrt{s_{N N}}=200 \mathrm{GeV}$ and $|y|<0.5$

$y_{a}$ and $y_{b}$ via the dependences $x_{1,2}=x_{1,2}\left(y_{a}, y_{b}\right)$ (see [5]) which include the fractal dimensions $\delta_{1}$ and $\delta_{2}$. The correlation between these quantities is reflected in symmetry properties of the function $\psi(z)$. The recoil mass $M_{X}$ reflects therefore an internal connection to the structure of the colliding objects, constituent interactions, and process of formation of the individual hadrons. 

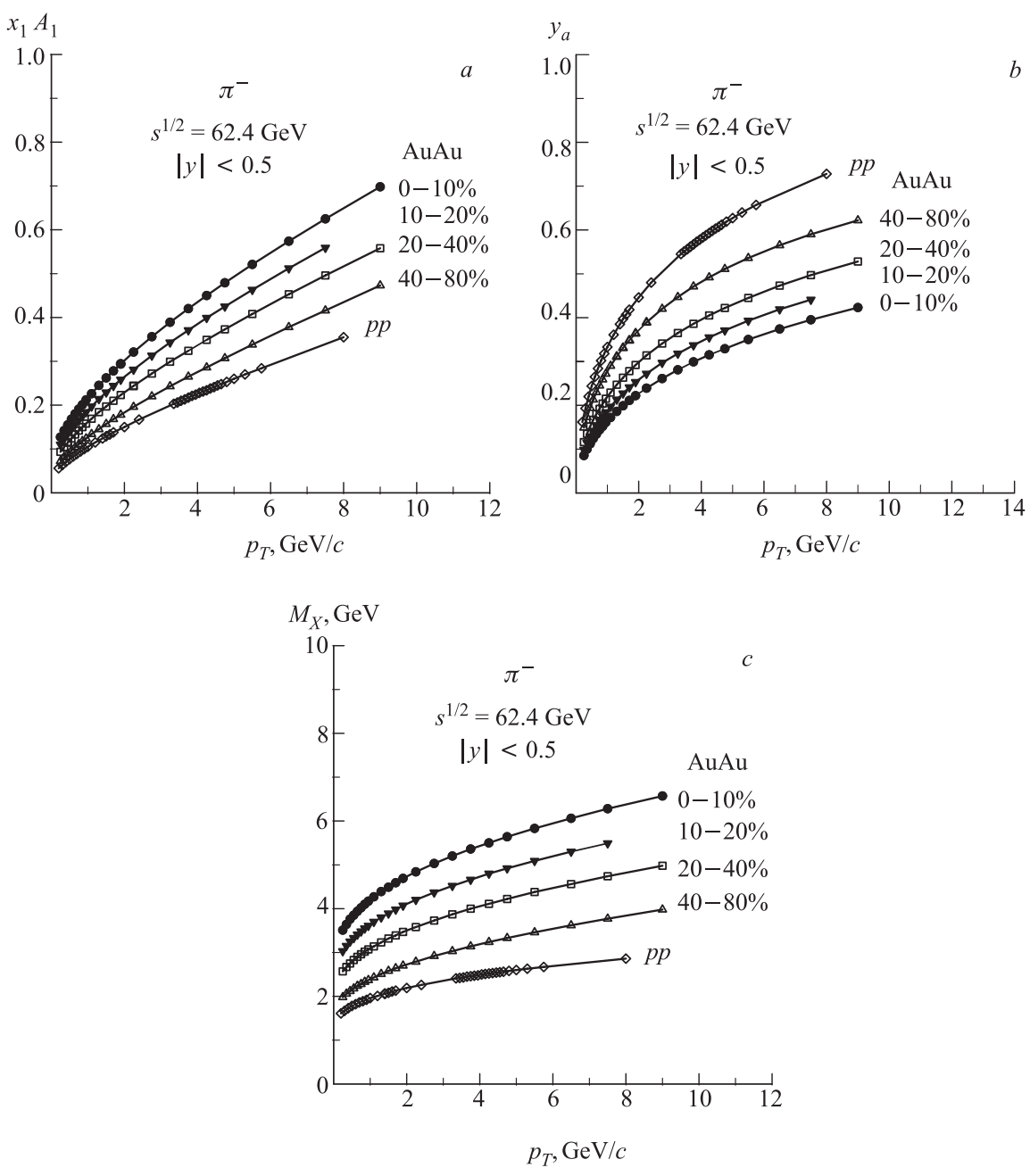

Fig. 10. The dependence of the fractions $A_{1} x_{1}(a), y_{a}(b)$, and the recoil mass $M_{X}(c)$ on the transverse momentum $p_{T}$ for $\pi^{-}$mesons produced in $p p$ and AuAu collisions at different centralities. The symbols correspond to data measured at $\sqrt{s_{N N}}=62.4 \mathrm{GeV}$ and $|y|<0.5$

Figures $9, c, 10, c, 11, c$, and 12, $c$ show the dependence of the recoil mass on the transverse momenta of the negative pions produced in the central rapidity region in $\mathrm{AuAu}$ and $\mathrm{PbPb}$ collisions at the energy $\sqrt{s}_{N N}=200,62.4$, 17.3, and $9.2 \mathrm{GeV}$. All curves demonstrate growth with $p_{T}$ which is followed at $\sqrt{s}_{N N}=62.4$ and $200 \mathrm{GeV}$ by a successive flattening. The dependences 

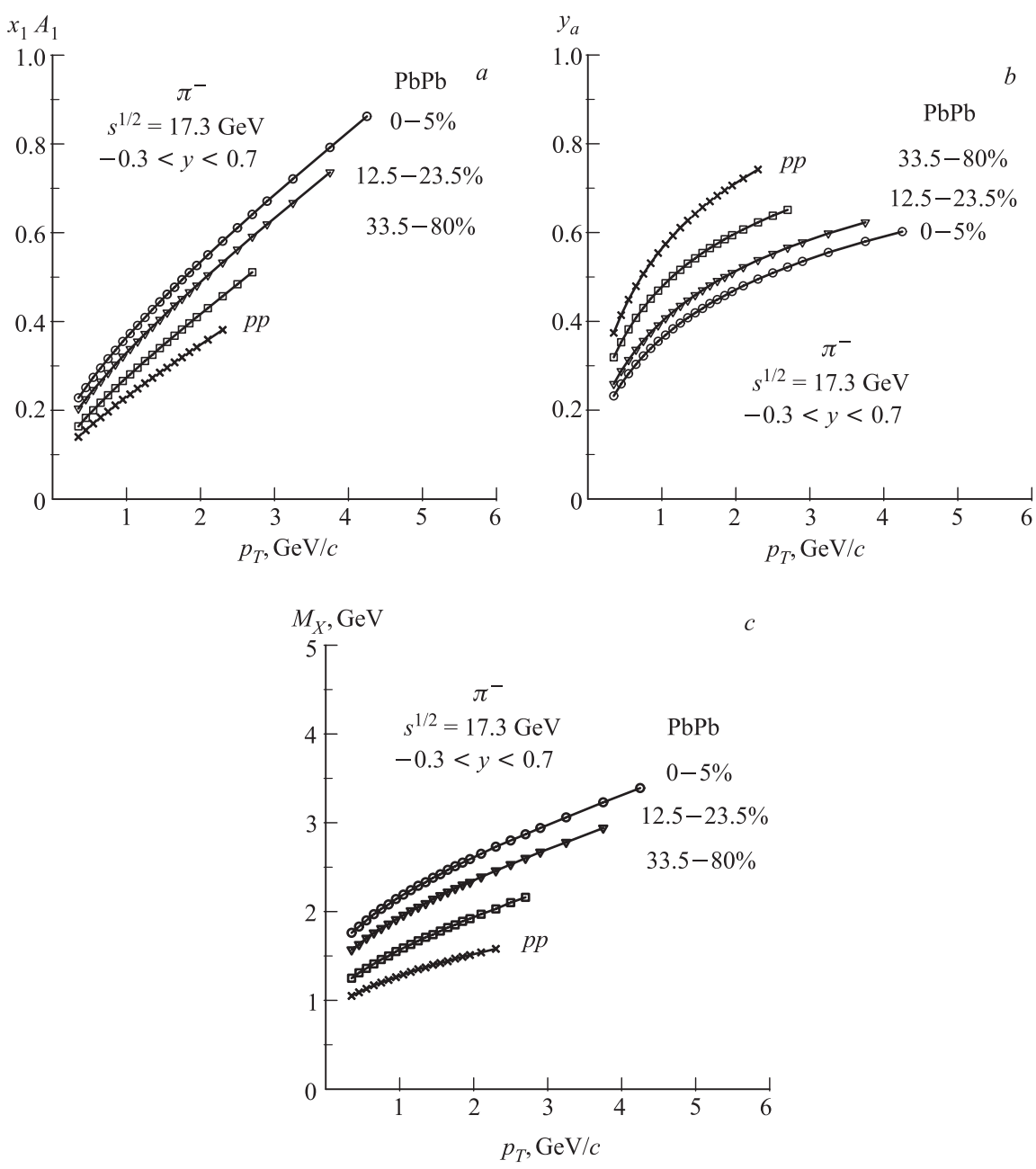

Fig. 11. The dependence of the fractions $A_{1} x_{1}(a), y_{a}(b)$ and the recoil mass $M_{X}(c)$ on the transverse momentum $p_{T}$ for $\pi^{-}$mesons produced in $p p$ and $\mathrm{PbPb}$ collisions at different centralities. The symbols correspond to data measured at $\sqrt{s_{N N}}=17.3 \mathrm{GeV}$ and $-0.3<y<0.7$

reveal a characteristic increase with the collision energy. The growth of $M_{X}$ with $p_{T}$ is larger for nucleus collisions in comparison with its slight increase for $p p$ interactions. The values of $M_{X}$ and their growth with $p_{T}$ become larger with the collision centrality. This means that the momentum balance in a subprocess underlying the pion production is compensated with growing number of particles 

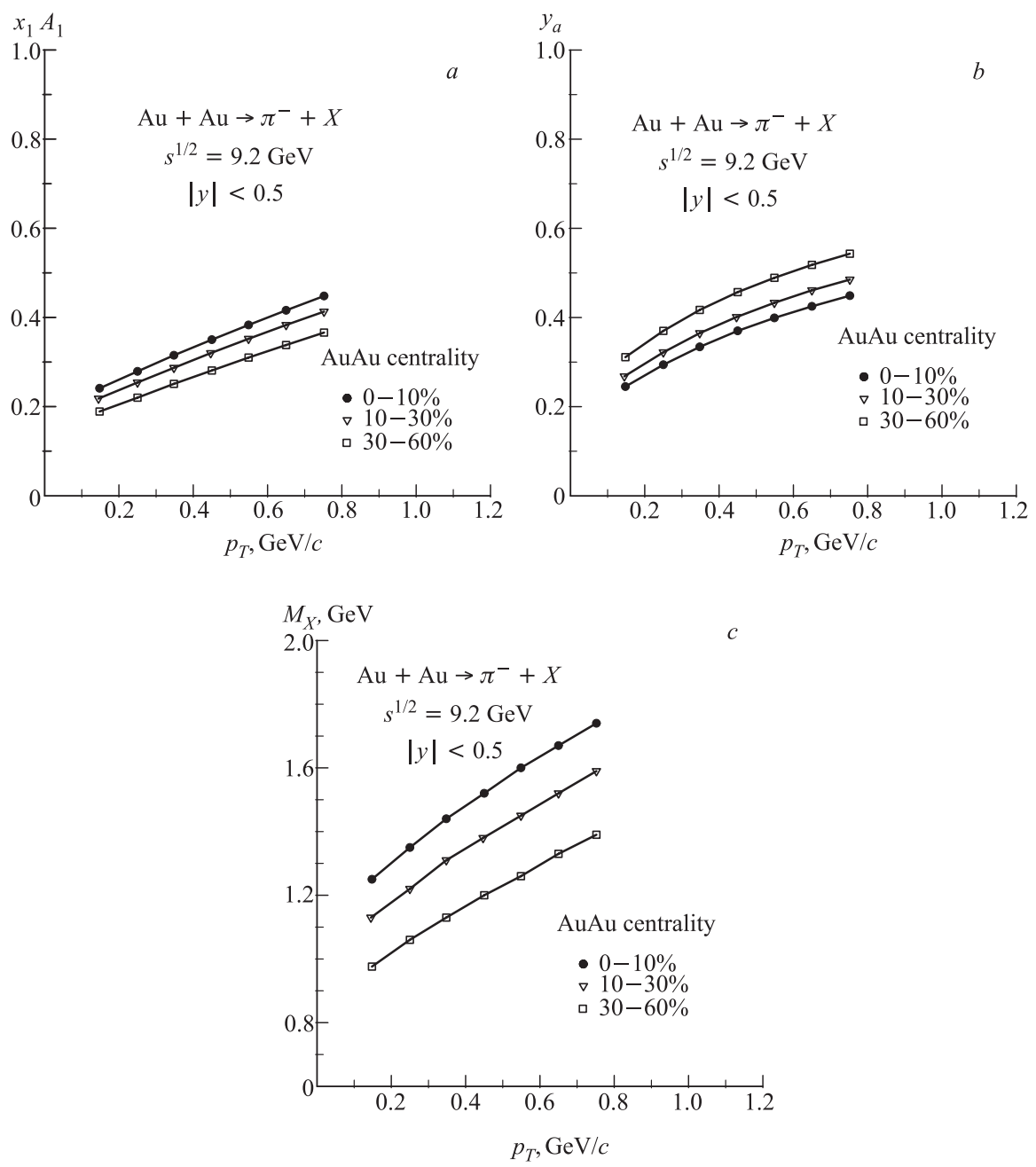

Fig. 12. The dependence of the fractions $A_{1} x_{1}(a), y_{a}(b)$, and the recoil mass $M_{X}(c)$ on the transverse momentum $p_{T}$ for $\pi^{-}$mesons produced in AuAu collisions at different centralities. The symbols correspond to data measured at $\sqrt{s_{N N}}=9.2 \mathrm{GeV}$ and $|y|<0.5$

moving in the away side direction when the centrality of the nuclear collisions increases. Considering an example of an inclusive pion with the momentum $p_{T}=4 \mathrm{GeV} / c$, the corresponding recoil masses $M_{X}$ in the central AuAu and 


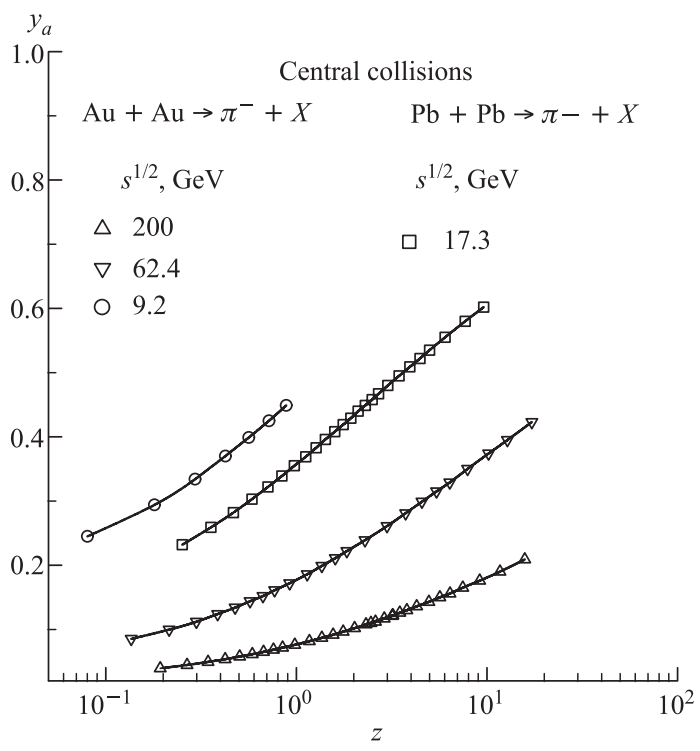

Fig. 13. The dependence of $y_{a}$ on $z$ for $\pi^{-}$mesons produced in central $\mathrm{AuAu}$ and $\mathrm{PbPb}$ collisions at $\sqrt{s}_{N N}=200,62.4,17.3$, and $9.2 \mathrm{GeV}$

$\mathrm{PbPb}$ collisions are equal to $3.5,5.5$, and $13 \mathrm{GeV}$ at the energy $\sqrt{s}_{N N}=17.3$, 62.4 , and $200 \mathrm{GeV}$, respectively.

6.3. Scale Dependence of Energy Losses. The developed microscopic scenario of hadron production in nucleus-nucleus collisions allows us to study the scale dependence of the energy losses. We would like to remind that the variable $z$ is scale-dependent quantity. It has properties of a fractal measure depending on the fractal dimensions $\delta$ and $\epsilon$. The energy losses by the creation of the inclusive particle are described in terms of the momentum fraction $y_{a}$. The dependence of $y_{a}$ on $z$ characterizes the energy losses at different scales. We assume that those scales where the energy losses are largest are most preferable for searching for a phase transition of nuclear matter. Figure 13 shows the scale dependence of $y_{a}$ (connected with energy loss) for central $A A$ collisions at $\sqrt{s}_{N N}=200,62.4$, 17.3, and $9.2 \mathrm{GeV}$. The energy loss decreases as the resolution with respect to the constituent subprocesses increases. At high $z$, a saturation of the scale density of energy loss $\left(d y_{a} / d z\right)$ for all energies is expected.

\section{CONCLUSIONS}

Experimental data on inclusive spectra of negative pions produced in $\mathrm{AuAu}$ collisions at RHIC energies $\sqrt{s}_{N N}=200,62.4$, and $9.2 \mathrm{GeV}$ were analyzed in 
the framework of $z$-scaling. The results are compared with the NA49 data on pion spectra measured in $p p$ and $\mathrm{PbPb}$ collisions at $\sqrt{s}_{N N}=17.3 \mathrm{GeV}$. The experimental data in $z$-presentation indicate similarity as a characteristic feature of mechanism of pion production. It was argued that this property includes structure of the colliding objects, interaction of their constituents, and character of the fragmentation process. A microscopic scenario of nucleus-nucleus interactions at a constituent level in terms of momentum fractions was developed. The $z$-presentation of the pion spectra in $\mathrm{AuAu}$ and $\mathrm{PbPb}$ collisions reveals scaling features identical with the $z$-scaling in the elementary collisions.

The parameter $c$ interpreted as «specific heat» of the medium produced in heavy ion collisions was established to decrease with the increasing system size, $c_{p p}=0.25>c_{d \mathrm{Au}}=0.23>c_{\mathrm{CuCu}}=0.14>c_{\mathrm{AuAu}}=0.11$. The values of $c$ are independent of the collision energy and centrality. The fractal dimension dependence $\epsilon_{A A}=\epsilon_{0}\left(d N_{\mathrm{ch}} / d \eta\right)+\epsilon_{p p}$ of the fragmentation process on collision centrality allows us to restore the unique shape of the scaling function for $p p$ and $A A$ collisions over a wide range of $z$. In the hard- $p_{T}$ region (large $z$ ), the scaling function manifests typical power behavior, $\psi(z) \simeq z^{-\beta}$, with a constant value of the slope parameter $\beta$. We have estimated the energy losses of the secondary partons passing through the medium created in the nuclear collisions. They depend on the collision energy, transverse momentum, and centrality. It was shown that energy loss increases in a specific manner with the collision energy and centrality, and decreases with $p_{T}$. The scale dependence of energy losses was studied. The obtained results may be exploited to search for and study of new physics phenomena in pion production in heavy ion collisions at U70, RHIC, LHC, and at the proposed projects NICA and FAIR.

Acknowledgement. The investigations have been supported by the IRP AVOZ10480505, by the Grant Agency of the Czech Republic under Contract No.202/07/0079 and by the special program of the Ministry of Science and Education of the Russian Federation, grant RNP.2.1.1.2512.

\section{REFERENCES}

1. Adams J. et al. (STAR Collab.) // Phys. Rev. Lett. 2003. V.91. P. 172302.

2. Arsene I. et al. (BRAHMS Collab.) // Phys. Rev. Lett. 2003. V.91. P. 172305.

3. Adler S. S. et al. (PHENIX Collab.) // Phys. Rev. C. 2004. V.69. P. 034910.

4. Back B. B. et al. (PHOBOS Collab.) // Phys. Rev. Lett. 2005. V.94. P. 082304.

5. Zborovský I., Tokarev M. V. // Phys. Rev. D. 2007. V.75. P. 094008.

6. Zborovský I., Tokarev M. V. // Int. J. Mod. Phys. A. 2009. V.24. P. 1. 
7. Tokarev M. V., Zborovský I. // Phys. At. Nucl. 2007. V.70. P. 1294.

8. Stavinsky V. S. // Sov. J. Part. Nucl. 1979. V.10. P. 949.

9. Antreasyan D. et al. // Phys. Rev. D. 1979. V.19. P. 764.

10. Alper B. et al. (BS Collab.) // Nucl. Phys. B. 1975. V. 100. P. 237.

11. Jaffe D. E. et al. // Phys. Rev. D. 1989. V.40. P. 2777.

12. Adams J. et al. (STAR Collab.) // Phys. Lett. B. 2006. V.637. P. 161.

13. Abelev B. I. et al. (STAR Collab.) // Phys. Rev. Lett. 2006. V.97. P. 152301.

14. Zborovský I., Tokarev M. V., Panebratsev Yu.A., Škoro G. P. // Phys. Rev. C. 1999. V.59. P. 2227.

15. Abelev B. I. et al. (STAR Collab.) // Phys. Lett. B. 2007. V.655. P. 104.

16. Alt C. et al. (NA49 Collab.) // Phys. Rev. C. 2008. V.77. P. 034906.

17. Kumar L. et al. (STAR Collab.) // Proc. of Strange Quark Matter 2008, Beijing, China, October 6-10, 2008; http://qm.phys.tsinghua.edu.cn/thu-henp/2008/sqm2008/; arXiv:0812.4099 [nucl-ex].

18. Adams J. et al. (STAR Collab.) // Phys. Lett. B. 2006. V.637. P. 161.

19. Alper B. et al. (BS Collab.) // Nucl. Phys. B. 1975. V. 100. P. 237.

20. Büsser F. W. et al. (CCRS Collab.) // Nucl. Phys. B. 1976. V. 106. P. 1. 


\section{Корректор T. Е. Попеко}

Подписано в печать 4.06.2009.

Формат $60 \times 90 / 16$. Бумага офсетная. Печать офсетная.

Усл. печ. л. 1,68. Уч.-изд. л. 2,26. Тираж 415 экз. Заказ № 56621.

Издательский отдел Объединенного института ядерных исследований 141980, г. Дубна, Московская обл., ул. Жолио-Кюри, 6.

E-mail: publish@jinr.ru www.jinr.ru/publish/ 